\title{
Giant Cell Tumor of the Lateral Skull Base: Diagnostic and Management Options
}

\author{
Nicholas T. Gamboa ${ }^{1}$ Brenden Ronna ${ }^{1}$ Christina T. Gamboa ${ }^{2}$ Cheryl A. Palmer ${ }^{3}$ Min S. Park \\ Richard K. Gurgel ${ }^{4}$ William T. Couldwell ${ }^{1} \quad$ M. Yashar S. Kalani ${ }^{1}$
}

\footnotetext{
1 Department of Neurosurgery, Clinical Neurosciences Center, University of Utah School of Medicine, Salt Lake City, Utah, United States

2 Department of Radiation Oncology, Stephenson Cancer Center, University of Oklahoma Health Sciences Center, Oklahoma City, Oklahoma, United States

${ }^{3}$ Division of Anatomic Pathology, Department of Pathology, University of Utah School of Medicine, Salt Lake City, Utah, United States

${ }^{4}$ Division of Otolaryngology, Department of Surgery, University of Utah School of Medicine, Salt Lake City, Utah, United States
}

Address for correspondence M. Yashar S. Kalani, MD, PhD, Department of Neurosurgery, University of Virginia, 7 Hospital Drive, Charlottesville, VA 22908, United States (e-mail: neuropub@hsc.utah.edu).

J Neurol Surg Rep 2018;79:e41-e54.
Abstract
Keywords
- giant cell tumor of bone
- lateral skull base tumor
- neurosurgery
- osteoclastoma
- radiotherapy

Giant cell tumor of bone (GCTB) is a rare, benign, osteolytic neoplasm that most commonly occurs in early adulthood and often involves the long bones of the body. Although GCTB largely affects the epiphyses of long bones, several reports of GCTB involvement of the cranial and facial bones exist in the literature. In addition to reviewing other reported cases of GCTBs of the lateral skull base in the literature, the authors report here on the clinical presentation, radiographic findings, and neurosurgical management of a patient found to have a GCTB of the middle and infratemporal fossae, which was treated by aggressive en bloc resection of the lateral skull base.

\section{Introduction}

Giant cell tumor of bone (GCTB; or osteoclastoma) is an uncommon, benign, osteolytic neoplasm. GCTB comprises 3 to $5 \%$ of all primary bone tumors and occurs predominantly in early adulthood (peak incidence ages, 20-40 years) with a slight female predominance $(3: 2) .^{1-3}$ These tumors are thought to originate from neoplastic nonosteogenic stromal cells of the bone marrow and are characterized histologically by numerous multinucleated osteoclastic giant cells diffusely distributed among a background of mononuclear stromal and macrophage lineage cells. ${ }^{1,4-6}$ GCTB most commonly affects the epiphyses of long bones, particularly of the distal femur and proximal tibia. ${ }^{6}$ Patients classically present with a combination of pain, swelling, or pathologic fracture at the tumor origin. ${ }^{7}$ Although regarded as benign, GCTB can recur locally following en bloc surgical resection. ${ }^{8,9}$ In 2 to $3 \%$ of cases, GCTBs can hematogenously metastasize to the lungs, resulting in benign pulmonary implants with rare malignant transformation. ${ }^{9,10}$ Despite an improved understanding of the molecular and cellular biology underlying the GCTB pathogenesis, the behavior of this tumor is often heterogeneous and can be difficult to predict on the basis of clinical, radiographic, or histologic features.

Although involvement of the appendicular skeleton is more typical for GCTB, axial skeleton involvement, especially of the cranial and facial bones, has also been reported and is becoming increasingly appreciated in the literature. Approximately $2 \%$ of GCTBs involve the head and neck. ${ }^{11-13}$ Involvement of the axial skeleton is often associated with increased morbidity because of local infiltration of critical structures and the associated difficulty of complete tumor resection, particularly compared with resection of GCTBs of the appendicular skeleton. ${ }^{8,14} \mathrm{GCTB}$ of the skull has also been reported to behave in a locally aggressive fashion. ${ }^{15}$ Nevertheless, surgery remains the treatment of choice for GCTBs, including those in the skull, received

July 3,2017 accepted after revision February 27, 2018
DOI https://doi.org/

10.1055/s-0038-1645885. ISSN 2193-6358.
@ 2018 Georg Thieme Verlag KG
Stuttgart · New York

License terms

(1) $\Theta \circledast$ 
with en bloc or wide local excision portending the lowest risk of recurrence and best clinical outcomes in patients. ${ }^{4,16}$ Although much research has focused on GCTBs involving the long bones, numerous studies have also reported on GCTBs involving the lateral skull base. Here, we review other reported cases of GCTBs involving the lateral skull base and report on the clinical presentation, radiographic findings, neurosurgical management, and outcome of a patient with a GCTB of the middle and infratemporal fossae.

\section{Methods}

A literature search was performed using the PubMed/Medline database for cases of GCTB of the lateral skull base. The literature search spanned articles published between 1970 and June 2017. The keywords utilized in the search included: "giant cell tumor of bone," "GCTB," "GCT," “osteoclastoma," "lateral skull base," and "skull base tumor." The list of publications was reviewed for articles with relevance to our study (i.e., reports of GCTB of the lateral skull base). Full-text articles in the English language were reviewed and chosen based on whether they included a reported case of a patient with a GCTB involving the lateral skull base.

\section{Case Report}

\section{History and Examination}

A 22-year-old man presented to the neurosurgical service after sustaining a traumatic brain injury when he was hit by a vehicle while riding his bike without a helmet. The patient experienced a brief loss of consciousness at the scene and reported a nonfluctuating headache. He was taken to an outside hospital, where a noncontrast head computed tomography (CT) scan demonstrated a left temporal intraparenchymal hemorrhage, left temporal bone fractures, multiple facial bone fractures, and an incidental lytic bone mass of the left lateral skull base (-Fig. 1). The patient was transferred to our institution for further evaluation.

Upon additional history gathering, the patient reported decreased hearing in the left ear over several months, as well as a recent slight bulging of his left face. On examination, the patient was awake, alert, and oriented to person and place. Cranial nerves were grossly intact, except for diminished sensorineural hearing in his left ear. Motor and sensory examinations were grossly intact throughout. He underwent diagnostic magnetic resonance imaging (MRI), which revealed a large tumor extending from the middle cranial fossa into the infratemporal fossa, with an associated intraparenchymal hemorrhage involving the left temporal lobe (-Fig. 2). Given this finding, the patient was taken to the operative theater for tumor resection.

\section{Operative and Postoperative Course}

The patient was positioned supine and secured in a Mayfield frame for microsurgical resection of the lesion. Given the infratemporal extension of the tumor, a Fisch-type approach was utilized, with a preauricular incision providing access to the infratemporal and intracranial components of this neoplasm. After elevation of the temporalis muscle, the tumor was found to be intimately involved with the bony structures. Frozen and eventual final pathology samples were consistent with a giant cell tumor without malignant features (-Fig. $\mathbf{3}$ ). Accordingly, a gross total resection of the tumor with negative margins was performed.

The temporal bone was drilled thoroughly with sacrifice of the left vestibulocochlear nerve to attain adequate margins. The left facial nerve was identified and skeletonized to protect and preserve function. Tumor-infiltrated temporal, frontal, and zygomatic bones were removed. Tumor feeding vessels arising from the superficial and deep temporal

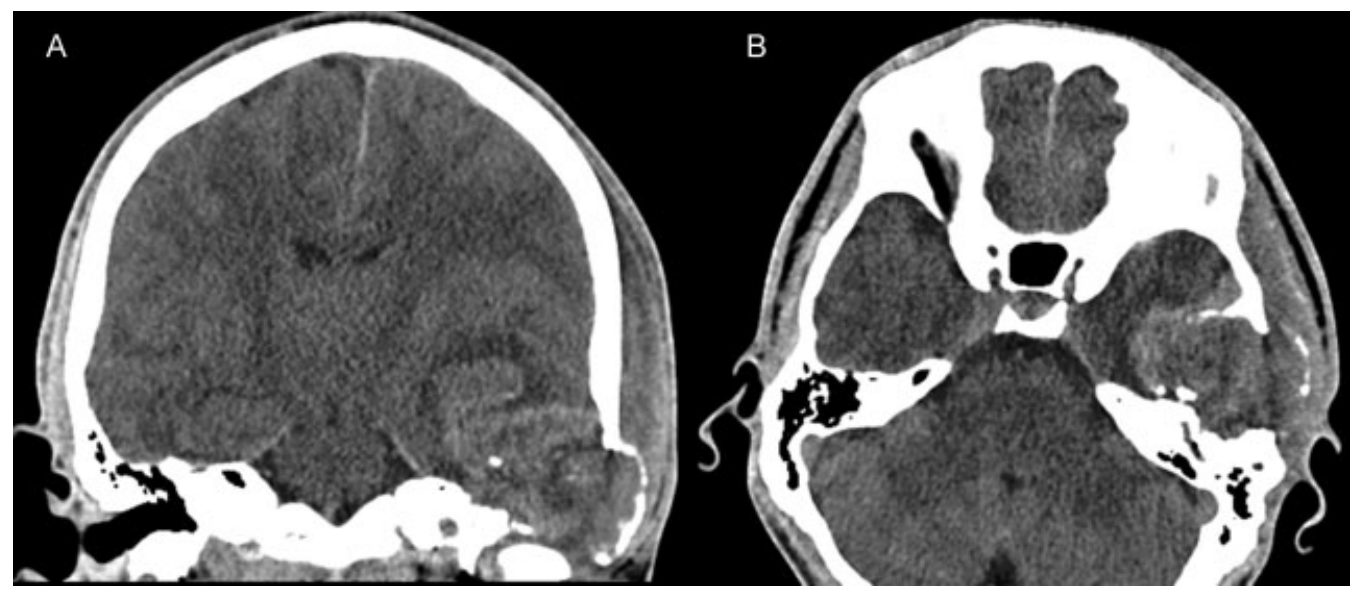

Fig. 1 Preoperative head computed tomography (CT) showing giant cell tumor of bone (GCTB) arising from the left lateral skull base. (A) Coronal head CT without contrast demonstrating a lytic mass of the left squamous part of the temporal bone with extension into the left sphenoid bone, mastoid air cells, and middle ear cavity. Imaging demonstrates destruction of the left lateral wall of the middle cranial fossa with bony fragment displacement laterally, medially, and inferiorly. The mass is also associated with intracranial hemorrhage and air within the hematoma that extends into the left temporal lobe. Moderate mass effect is present with left-to-right shift. (B) Axial head CT without contrast showing a lytic mass originating from the squamous part of the temporal bone. Imaging also reveals comminuted left orbital fractures, left maxillary sinus fractures, and a left zygomatic arch fracture. 


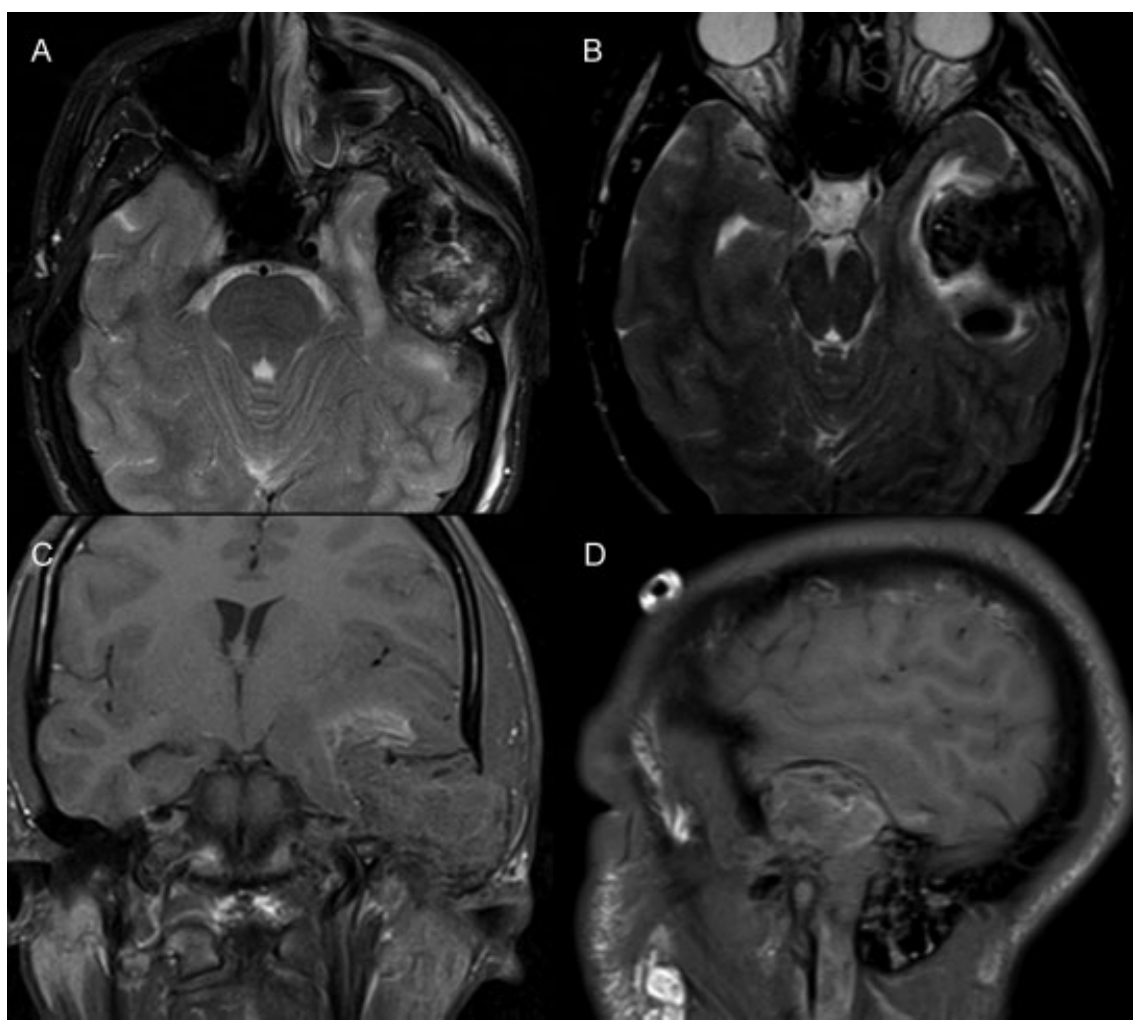

Fig. 2 Preoperative magnetic resonance imaging (MRI) of giant cell tumor of bone (GCTB) arising from the left lateral skull base. (A) Axial T2-weighted MRI demonstrating destructive heterogeneous, hypointense, peripherally enhancing lesion centered in the left squamous part of the temporal bone that measures $4.5 \times 4.0 \times 4.9 \mathrm{~cm}$. (B) Axial T2-weighted MRI demonstrating hypointense mass with extension anteriorly into the left sphenoid bone. Imaging is notable for intracranial extension, mass effect on the left temporal lobe, and surrounding vasogenic edema. Midline shift of $\sim 5 \mathrm{~mm}$ is present with early left uncal herniation, effacement of the left ambient cistern, and compression of the midbrain. (C) Coronal T1-weighted MRI demonstrating a mass arising from the squamous part of the temporal bone and extending into the left temporal lobe. (D) Sagittal T1-weighted MRI demonstrating anteroposterior and craniocaudal extension of the tumor.
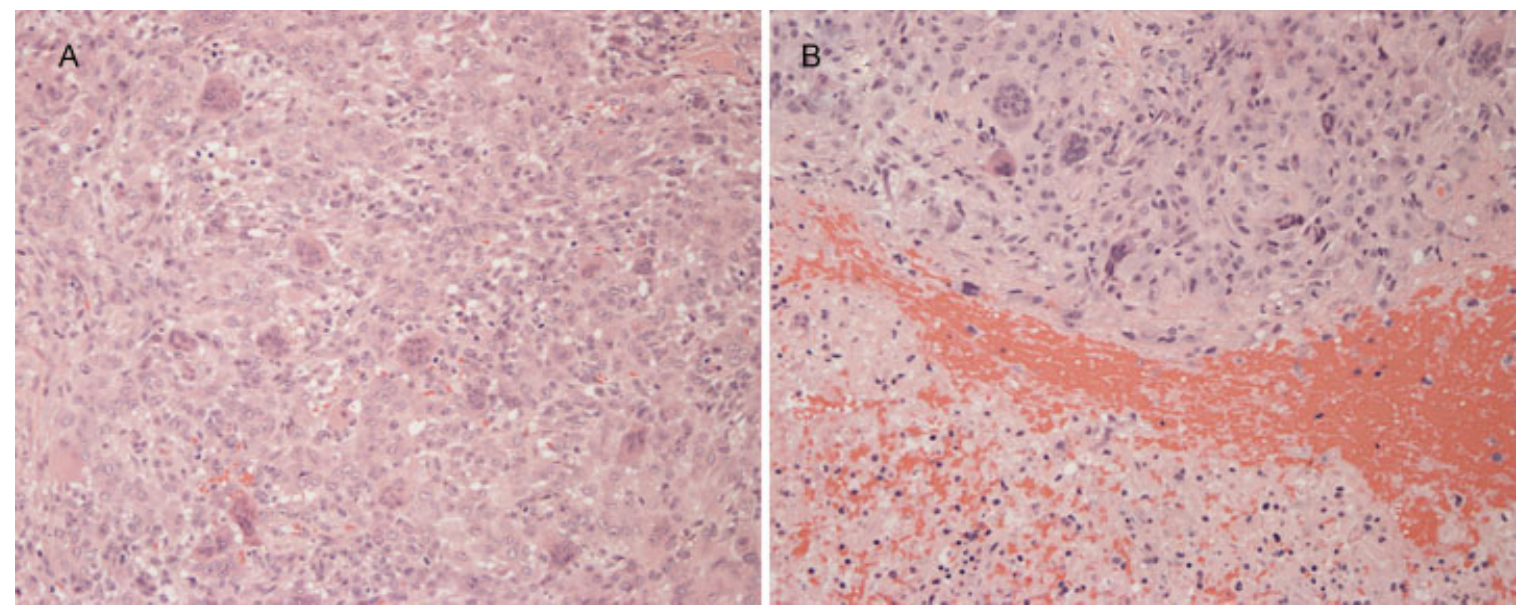

Fig. 3 Photomicrograph of hematoxylin and eosin (H\&E)-stained histopathologic specimens from a patient with giant cell tumor of bone (GCTB) of the infratemporal fossa. (A) H\&E stained specimen $(100 \times)$ demonstrating numerous multinucleated osteoclastic giant cells distributed diffusely among a background of neoplastic mononuclear stromal cells and mononuclear macrophage lineage cells. (B) H\&E stained specimen $(100 \times)$ demonstrating tumor necrosis with nearby multinucleated osteoclastic giant cells and mononuclear stroma cells.

arteries were coagulated to devascularize the tumor. Normal bony margins were obtained. With respect to the intracranial compartment, tumor involving the dura was resected. In addition, upon reflection of the dura, the tumor was found to involve the left lateral temporal lobe, causing hemorrhage and mass effect. The left temporal lobe involved by the tumor was surgically resected.

At the completion of the procedure, the facial nerve was stimulated to ensure its integrity, and meticulous hemostasis was obtained. To repair the skull base defect, mesh 


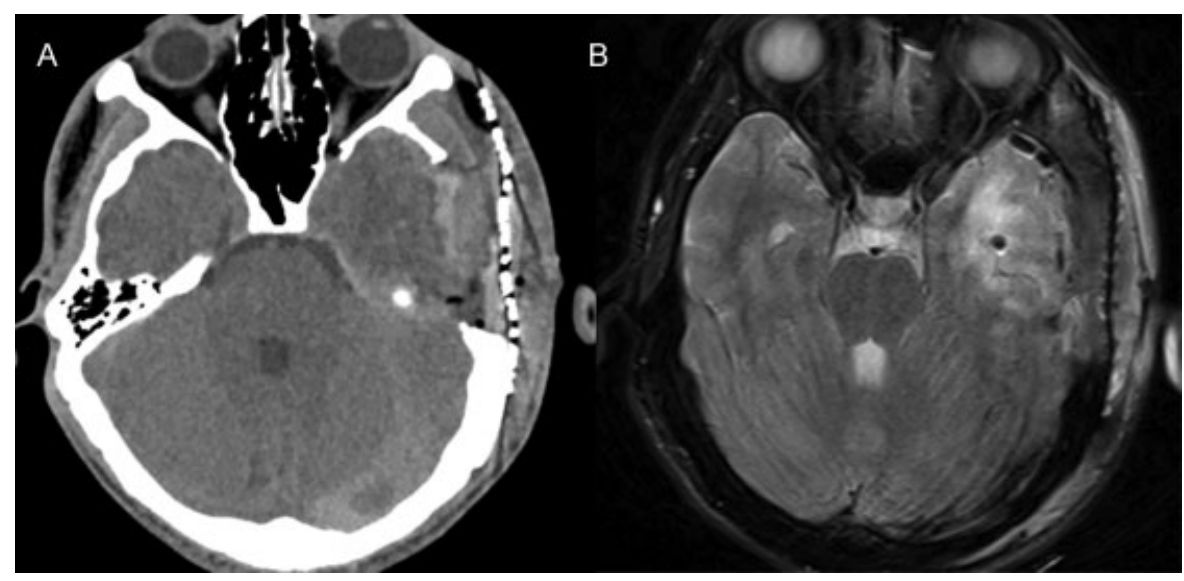

Fig. 4 Postoperative computed tomography $(\mathrm{CT})$ and magnetic resonance imaging (MRI) demonstrating gross total resection of giant cell tumor of bone (GCTB) of the left lateral skull base with mesh cranioplasty. (A) Axial head CT without contrast demonstrating postsurgical changes. Comminuted left orbital and maxillary sinus fractures are present. (B) Axial T2-weighted MRI demonstrating postsurgical changes from left temporal bone craniectomy, tumor resection, and mesh cranioplasty. Imaging shows no signs of residual disease. Complete surgical resection with resultant decompression and normalization of midline shift is apparent on postoperative imaging.

cranioplasty with polymethyl methacrylate was fashioned to a normal cranial contour. A small amount of superficial fat was placed into the site of the defect. MRI demonstrated complete removal of the tumor in this patient ( - Fig. 4). Postoperatively, the patient had nonserviceable hearing in the left ear, but was otherwise neurologically intact, including preservation of the facial nerve function, and was discharged home on postoperative day 5 . No adjuvant radiation was planned. At the 3-month follow-up, the patient was neurologically intact but did require cerebrospinal fluid diversion for a hygroma.

\section{Results}

- Table 1 summarizes the cases of tumors involving the lateral skull base identified in our review of the English language literature. Including our case, a total of 94 patients with GCTBs of the lateral skull base were identified through our review. As an aggregate, $56 \%$ of patients were male (53/94), while $44 \%$ were female (41/94). The mean age at presentation was 36.8 years (range: 0.17-79 years). Clinical presentations were variable and included headache $(33 \%, 29 / 88)$, hearing loss (31\%, 27/88), facial/preauricular swelling (22\%, 19/88), facial/ preauricular pain $(17 \%, 15 / 88)$, tinnitus (15\%, 13/88), aural fullness $(10 \%, 9 / 88)$, diplopia $(10 \%, 9 / 88)$, vision loss $(10 \%, 9 / 88)$, ear pain $(8 \%, 7 / 88)$, facial nerve palsy $(8 \%, 7 / 88)$, proptosis $(5 \%, 4 / 88)$, and dizziness $(5 \%, 4 / 88)$. GCTB origin, in order of frequency, was temporal bones (62\%, 58/94), sphenoid bones (32\%,30/94), occipital bones (5\%, 5/94), and frontal bones $(1 \%, 1 / 94)$.

Treatment data were reported for 91 patients. Of these patients, 52\% (47/91) received gross total resections, $46 \%$ (42/ 91) subtotal resections, and $2 \%(2 / 91)$ did not receive treatment. Of 90 patients with clear documentation, 37\% (33/90) received adjuvant radiotherapy following surgical treatment.

Of patients with reported follow-up, 14\% (12/86) had local disease recurrence. In addition, of those patients with recurrence, 50\% (6/12) had received subtotal resections alone, 25\% (3/12) received subtotal resections with adjuvant radiother- apy, and 25\% (3/12) received gross total resections alone. Of note, no patients receiving gross total resections with adjuvant radiotherapy developed recurrence $(n=4)$.

\section{Discussion}

GCTB is considered a benign, but locally aggressive, neoplasm of bone. These tumors are thought to originate from nonosteogenic neoplastic stromal cells of the bone marrow admixed with multinucleated osteoclastic giant cells. ${ }^{1,6}$ GCTBs most commonly affect the epiphyses of long bones, but have also been appreciated in the cranial and facial bones, including the skull base. ${ }^{6}$ Although considered a benign lesion, GCTB has a variable natural history, with the risk of local recurrence or distant metastasis being highly unpredictable. Unlike other types of cancer, metastatic spread of GCTB does not carry similar prognostic implications. ${ }^{17}$ Despite improved understanding of the underlying cellular and molecular biology underpinning GCTB pathogenesis, our knowledge regarding this tumor's behavioral heterogeneity remains incomplete.

GCTBs of the cranial and facial bones most commonly affect the temporal and sphenoid bones. ${ }^{14,15,18-20}$ GCTBs of the lateral skull base are often locally aggressive and can invade nearby critical structures. ${ }^{18,21}$ It is believed that these tumors arise in these areas because the bones of the mandible, sphenoid, ethmoid, and parts of the temporal bone form largely through the process of endochondral ossification. ${ }^{22}$ In contrast, the other cranial bones (i.e., frontal and parietal bones) arise from intramembranous ossification and are less frequently affected by GCTB. ${ }^{15,22}$ Patients with GCTB of the temporal bone typically present with headache, conductive hearing loss, aural fullness, preauricular pain, or facial weakness. ${ }^{18,20,23}$ In comparison, patients with GCTB of the sphenoid bone may present with symptoms such as headache, facial hypoesthesia, diplopia, blindness, or visual field defects. ${ }^{18,19}$

Both CT and MRI are used to identify and characterize GCTB of the lateral skull base. On CT imaging, GCTBs usually 
Giant Cell Tumor of the Lateral Skull Base Gamboa et al. e45

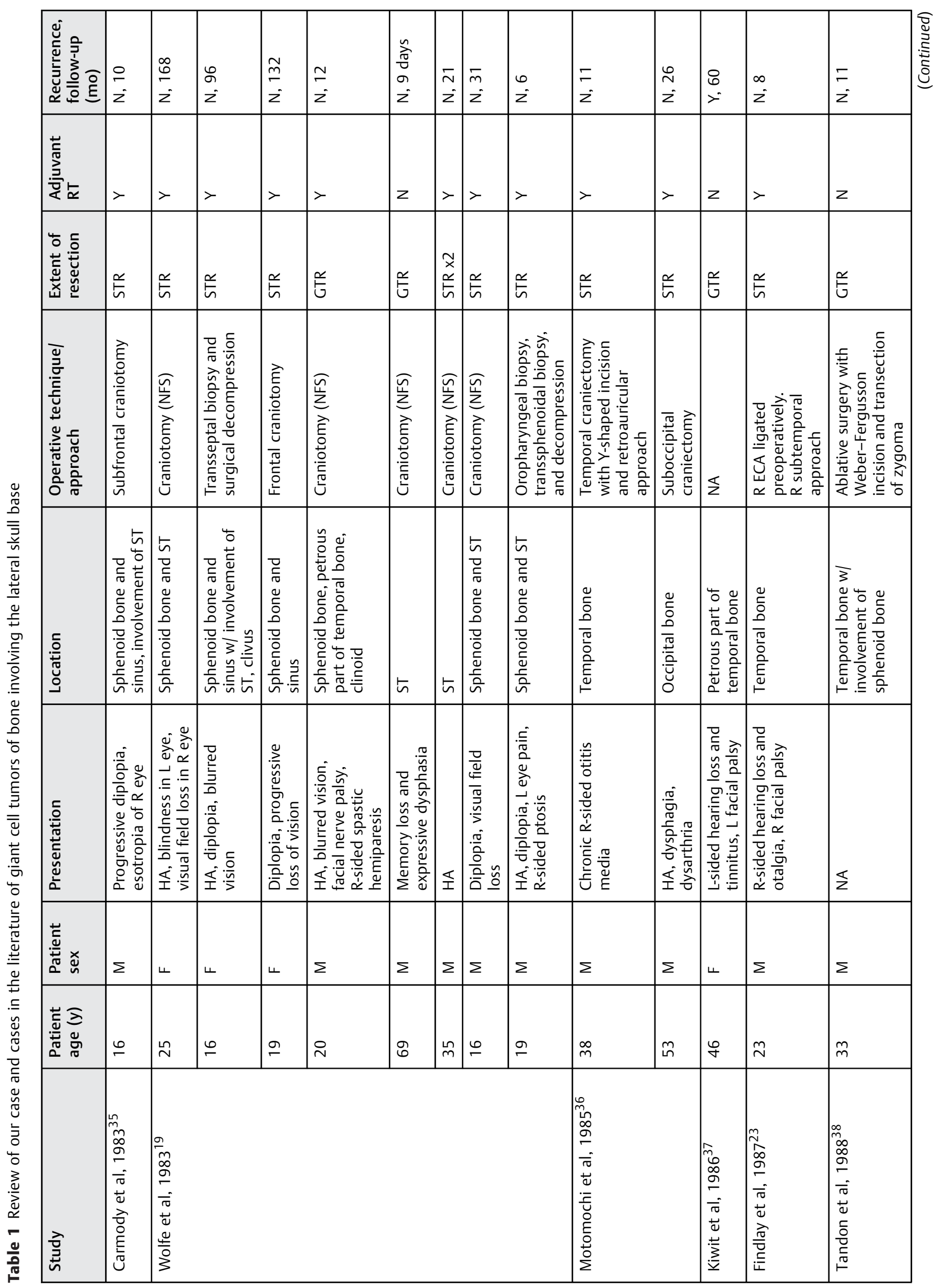




\begin{tabular}{|c|c|c|c|c|c|c|c|c|c|c|c|c|}
\hline 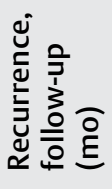 & $\dot{m}$ & $\mid \begin{array}{l}\stackrel{D}{1} \\
z \\
z\end{array}$ & $\stackrel{\bar{m}}{亠^{\prime}}$ & $\begin{array}{l}\infty \\
i \\
z\end{array}$ & $\begin{array}{l}\ddagger \\
\infty \\
z\end{array}$ & $\mid \begin{array}{l}\infty \\
\succ \\
\succ\end{array}$ & 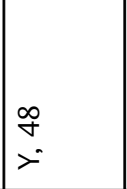 & $\begin{array}{l}\stackrel{\infty}{+} \\
z\end{array}$ & $\begin{array}{l}0 \\
z\end{array}$ & $\begin{array}{l}m \\
m \\
z\end{array}$ & $\Sigma$ & $\begin{array}{l}\dot{y} \\
m \\
z\end{array}$ \\
\hline 崩 & $>$ & $>$ & $z$ & $z$ & $>$ & $z$ & $z$ & $>$ & $z$ & $z$ & $z$ & $\S$ \\
\hline 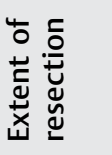 & 絁 & $\frac{a}{E}$ & 总 & 絁 & 兴 & 兴 & 号 & 妥 & 总 & 絔 & 亭 & $\Sigma$ \\
\hline 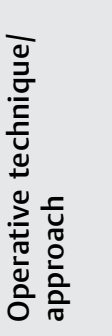 & $\Sigma$ & $\Sigma$ & $\Sigma$ & $\Sigma$ & $\Sigma$ & $\Sigma$ & $\Sigma$ & 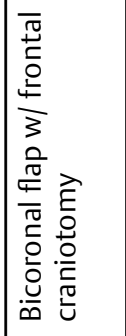 & 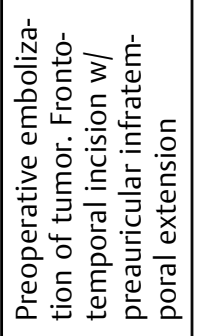 & 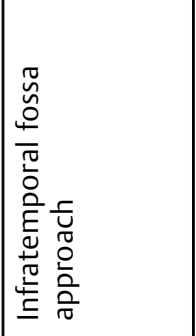 & 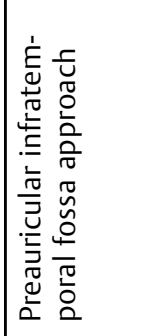 & 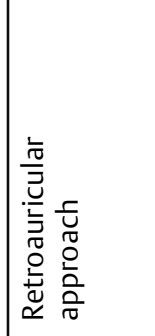 \\
\hline 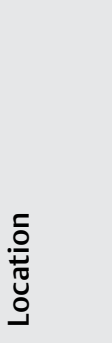 & 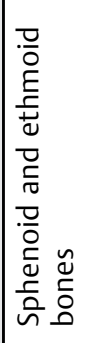 & 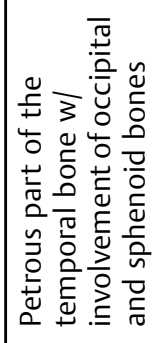 & 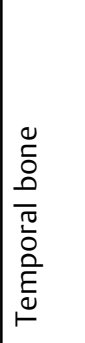 & 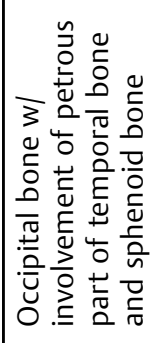 & $\mid$ & 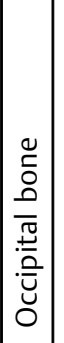 & 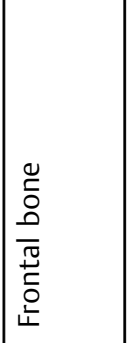 & 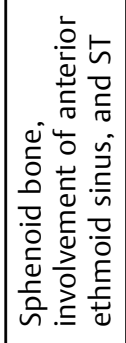 & 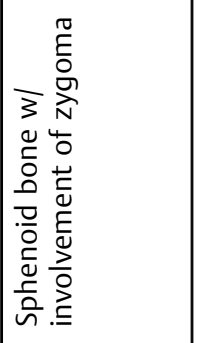 & 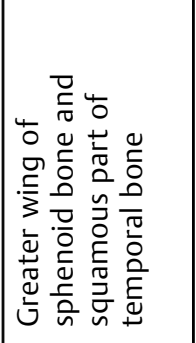 & 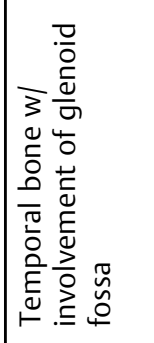 & 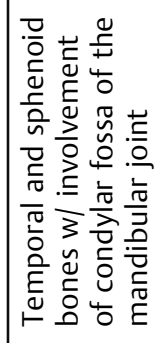 \\
\hline 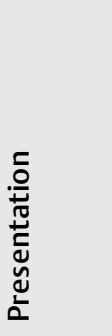 & 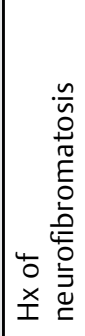 & 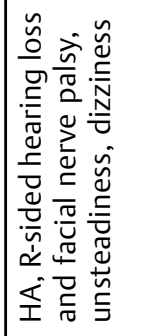 & 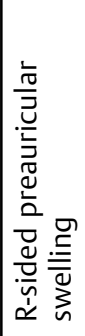 & \ & $\Sigma$ & $\mid$ & 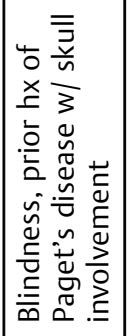 & 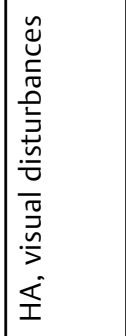 & 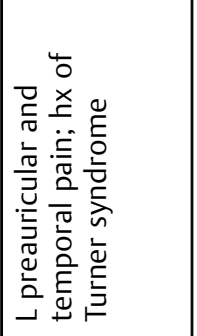 & 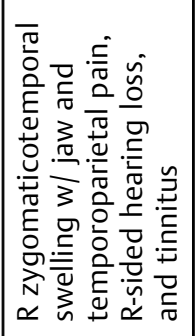 & 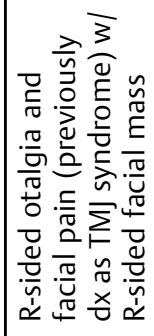 & \begin{tabular}{|l}
$\Sigma$ \\
$z$
\end{tabular} \\
\hline 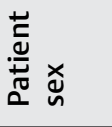 & $\sqcup$ & 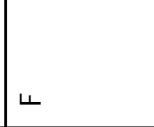 & 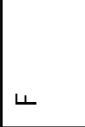 & $\sqcup$ & $\sqcup$ & $\bullet$ & 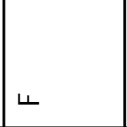 & $\sqcup$ & $\sqcup$ & $\Sigma$ & $\sqcup$ & ᄂ \\
\hline 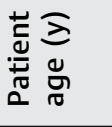 & $\tilde{\varphi}$ & 6 & $\infty$ & $\stackrel{\Xi}{\sim}$ & $\stackrel{\infty}{\sim}$ & 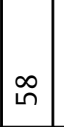 & $\stackrel{\infty}{\wedge}$ & $\stackrel{\nabla}{\leftarrow}$ & $\tilde{m}$ & $\stackrel{\varphi}{m}$ & 迤 & g \\
\hline 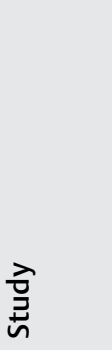 & 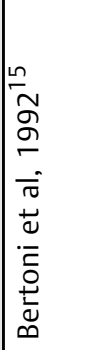 & & & & & & & 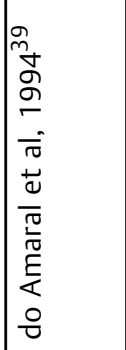 & 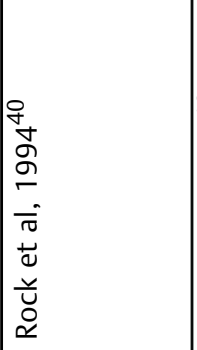 & 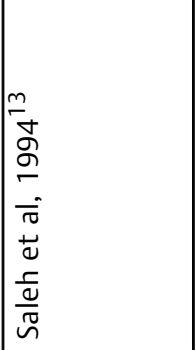 & 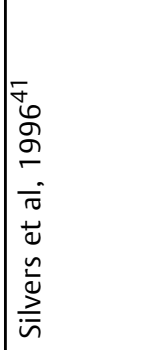 & 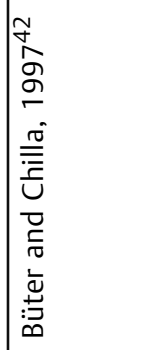 \\
\hline
\end{tabular}


Giant Cell Tumor of the Lateral Skull Base Gamboa et al. e47

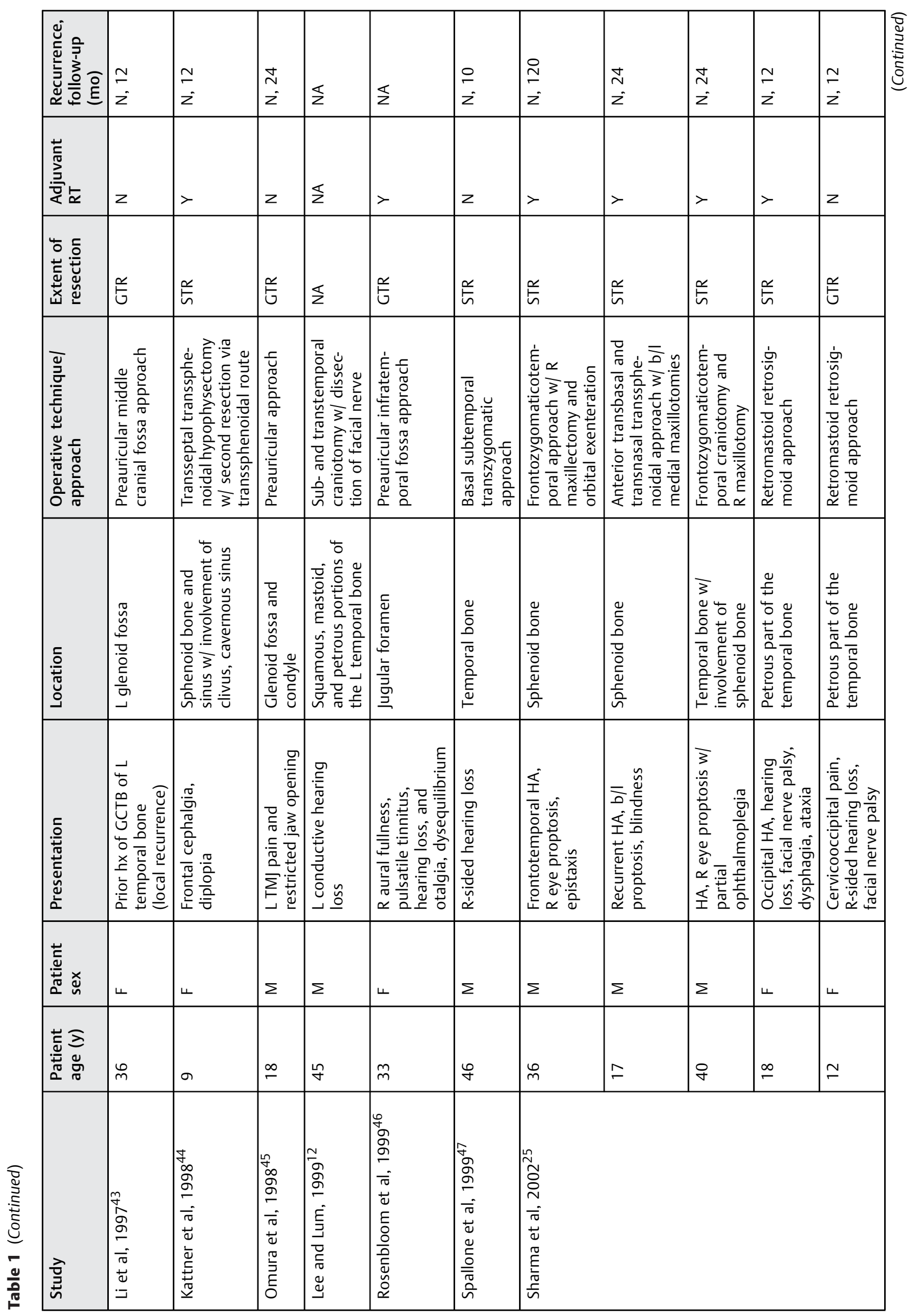




\begin{tabular}{|c|c|c|c|c|c|c|c|c|c|c|c|}
\hline 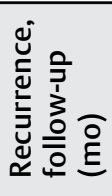 & \& & $\bar{z}$ & $\Sigma$ & 乏 & $\mid \begin{array}{l}\stackrel{N}{z} \\
z z\end{array}$ & $\begin{array}{l}\stackrel{\searrow}{ } \\
z\end{array}$ & $\begin{array}{l}\stackrel{5}{5} \\
>\end{array}$ & $\mid \begin{array}{l}m \\
z z\end{array}$ & $\mid \begin{array}{l}\bar{z} \\
z\end{array}$ & $\mid \begin{array}{l}\sim \\
z\end{array}$ & $\mid \begin{array}{l}\stackrel{N}{z} \\
z\end{array}$ \\
\hline 崩 & $>$ & $z$ & $z$ & $>$ & $z$ & $z$ & $>$ & $z$ & $z$ & $>$ & $z$ \\
\hline 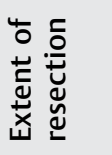 & $\mid \begin{array}{l}0 \\
\tilde{\Sigma} \\
z\end{array}$ & $\begin{array}{l}\text { U. } \\
\text { o } \\
z\end{array}$ & 舀 & $\frac{a}{\tilde{n}}$ & 兴 & 亭 & $\frac{a}{n}$ & 兴 & 旁 & $\frac{a}{n}$ & $\frac{a}{\underline{n}}$ \\
\hline 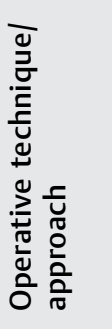 & 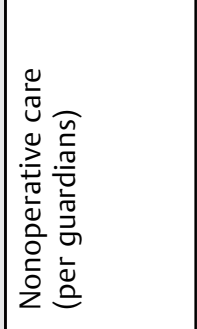 & 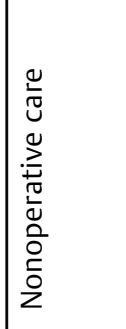 & 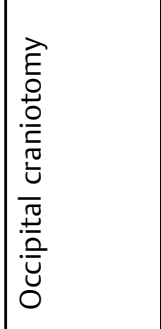 & 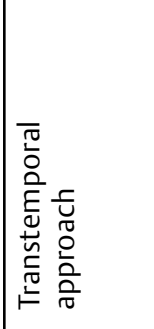 & 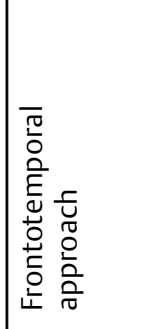 & 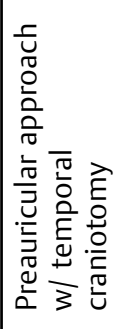 & 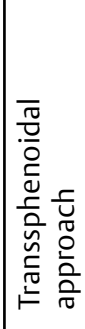 & 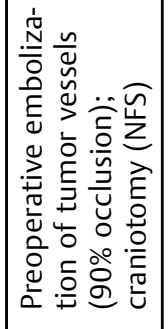 & 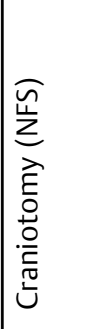 & 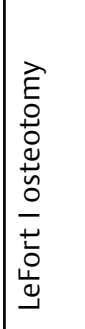 & 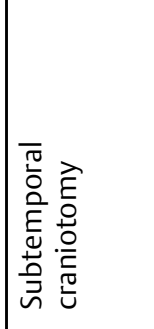 \\
\hline סั & 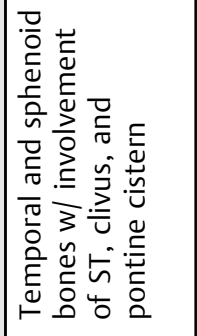 & 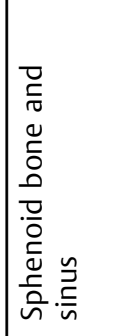 & 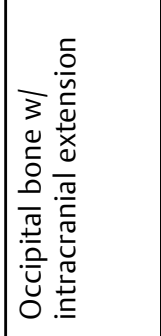 & 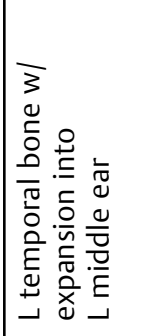 & 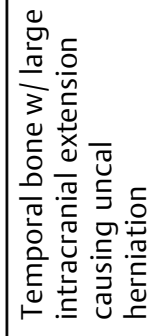 & 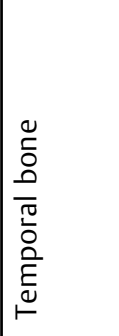 & 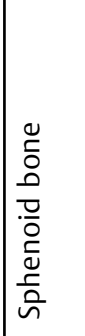 & 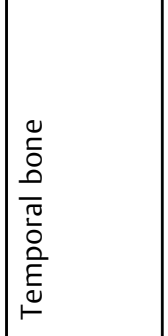 & 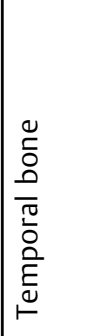 & 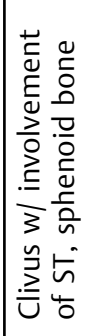 & 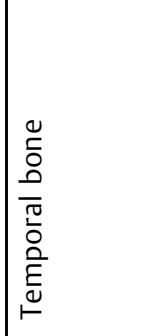 \\
\hline 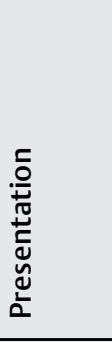 & 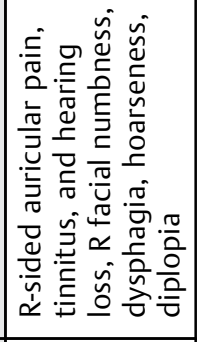 & 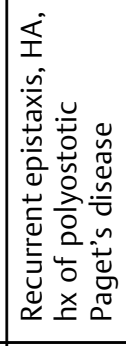 & 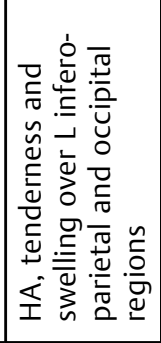 & 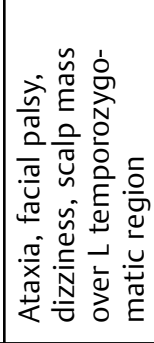 & 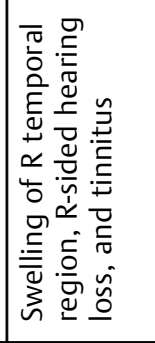 & 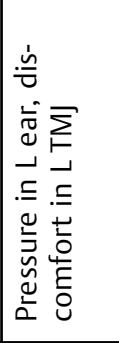 & 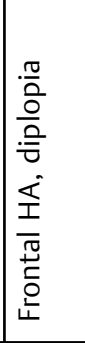 & 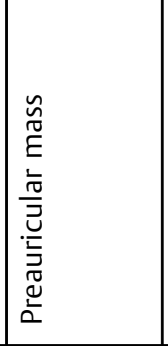 & 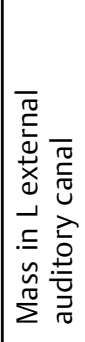 & 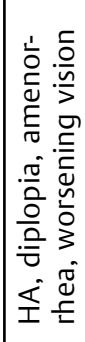 & 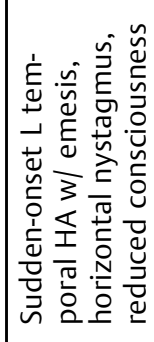 \\
\hline 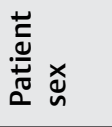 & 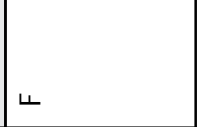 & $\Sigma$ & $\sqcup$ & 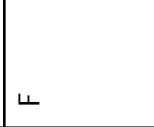 & $\Sigma$ & 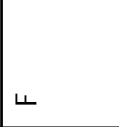 & 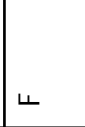 & $\sqcup$ & 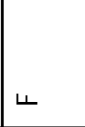 & 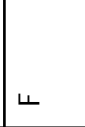 & 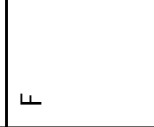 \\
\hline 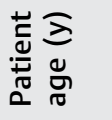 & $\infty$ & $\curvearrowright$ & $\stackrel{ \pm}{\sim}$ & $\overline{6}$ & $\stackrel{\wp}{\sim}$ & రే & 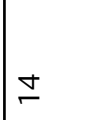 & $\sim$ & $\stackrel{m}{\tilde{0}}$ & $\approx$ & 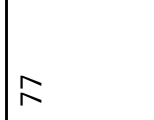 \\
\hline 咅 & 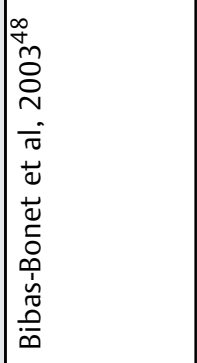 & 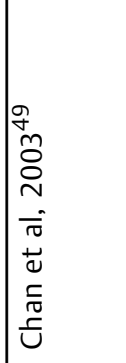 & 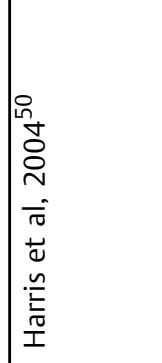 & 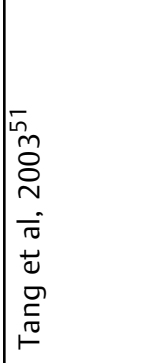 & 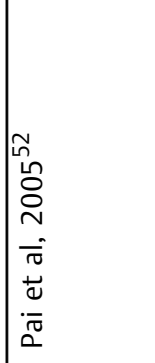 & 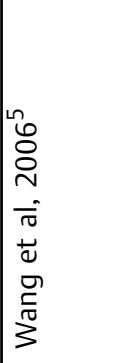 & 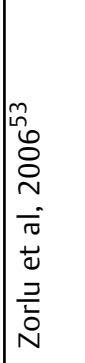 & 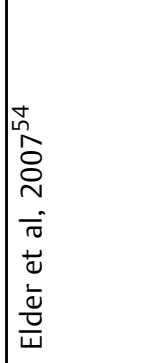 & & 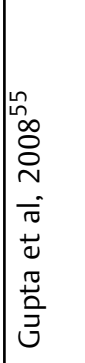 & 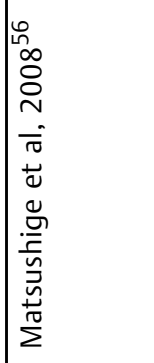 \\
\hline
\end{tabular}




\begin{tabular}{|c|c|c|c|c|c|c|c|c|c|c|c|c|c|c|c|c|c|c|c|c|c|c|}
\hline 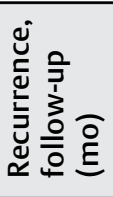 & $\begin{array}{l}0 \\
m \\
z\end{array}$ & $\begin{array}{l}0 \\
m \\
z \\
z\end{array}$ & $\mid \begin{array}{l}\stackrel{ }{\check{1}} \\
z ̇\end{array}$ & $\begin{array}{l}n \\
\dot{z}\end{array}$ & $\mid \begin{array}{l}0 \\
z\end{array}$ & $\begin{array}{l}\stackrel{\infty}{+} \\
z \\
z\end{array}$ & $\begin{array}{l}\simeq \\
z\end{array}$ & $\begin{array}{l}\bar{N} \\
z\end{array}$ & \begin{tabular}{l}
$\sigma$ \\
\multicolumn{1}{c}{}
\end{tabular} & 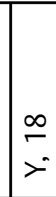 & $\begin{array}{l}\tilde{m} \\
z\end{array}$ & $\mid \begin{array}{l}\hat{N} \\
z \\
z\end{array}$ & $\begin{array}{l}n \\
z\end{array}$ & $\begin{array}{l}\stackrel{N}{N} \\
z \\
z\end{array}$ & $\left|\begin{array}{l}\tilde{m} \\
z \\
z\end{array}\right|$ & $\begin{array}{l}\bar{m} \\
z\end{array}$ & \begin{tabular}{l|l}
$m$ & \\
$z^{-}$ &
\end{tabular} & \begin{tabular}{l|l}
0 & 0 \\
+ & 1 \\
$>^{\prime}$ & $=$
\end{tabular} & \begin{tabular}{l|l}
$\curvearrowright$ & I \\
$z^{\prime}$ & $z$
\end{tabular} & \begin{tabular}{l|l}
$g$ & \\
$z$ & $\sim$ \\
$z$
\end{tabular} & $\Delta$ & \\
\hline 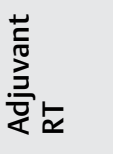 & $z$ & $z$ & $z$ & $>$ & $z$ & $z$ & $z$ & $>$ & $z$ & $z$ & $>$ & $>$ & $z$ & $>$ & $>$ & $z$ & $>\quad z$ & $z \mid z$ & $z z$ & $z \mid z$ & 18 & \\
\hline 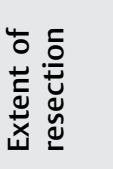 & $\Sigma$ & 兰 & 受 & $\frac{a}{n}$ & 兴 & 㤐 & 亭 & 号 & 亮 & $\stackrel{\underline{E}}{n}$ & 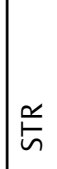 & $\stackrel{\underline{a}}{\sim}$ & 厸 & $\stackrel{\underline{a}}{\underline{s}}$ & 兴 & $\underline{\underline{G}}$ & $\stackrel{c}{\sim}$ & $\stackrel{ }{\sim}$ & 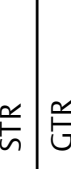 & 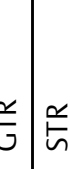 & 象 & \\
\hline 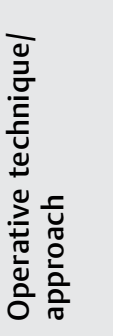 & 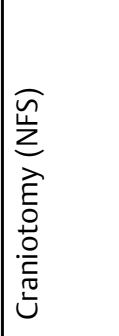 & 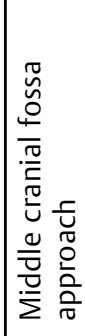 & 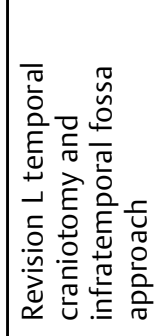 & ¿ & 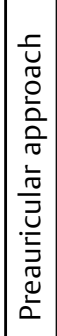 & 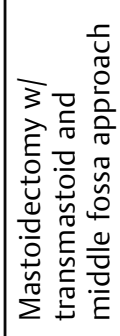 & 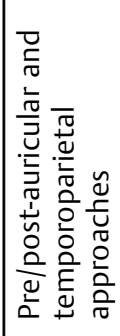 & ¿ & $\Sigma$ & z & $\Sigma$ & $\Sigma$ & $\Sigma$ & $\Sigma$ & $\Sigma$ & $\Sigma$ & $\Sigma$ & $\Sigma \leq$ & 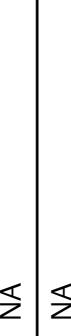 & $\Sigma \mid \Sigma$ & 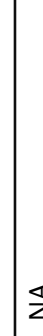 & \\
\hline ס. & 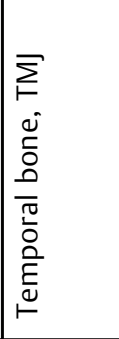 & 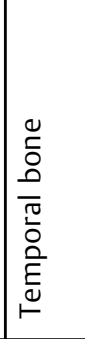 & 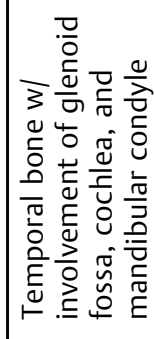 & 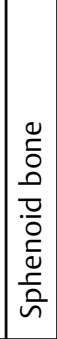 & 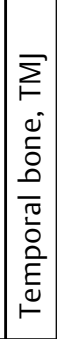 & 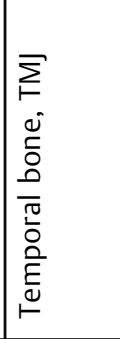 & 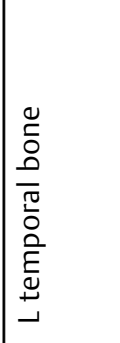 & 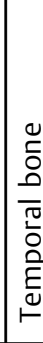 & 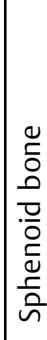 & 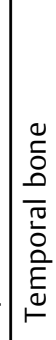 & 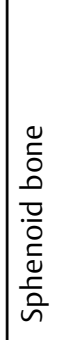 & 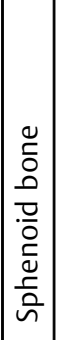 & 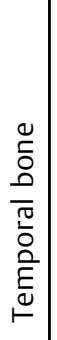 & 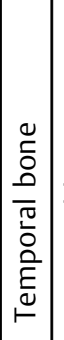 & 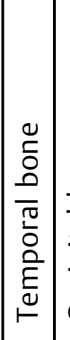 & 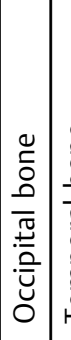 & 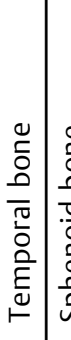 & 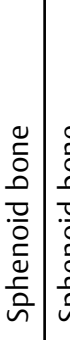 & 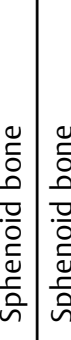 & 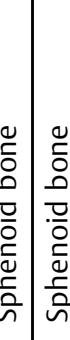 & 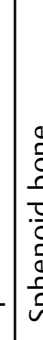 & \\
\hline 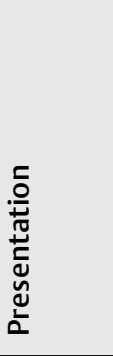 & 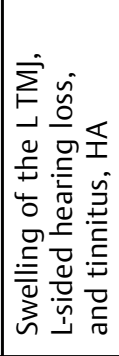 & 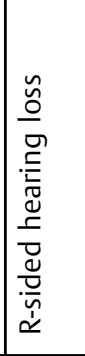 & 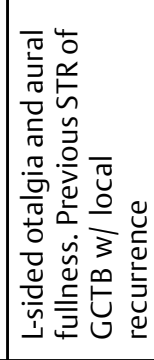 & £ & 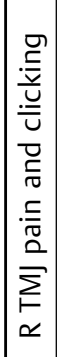 & 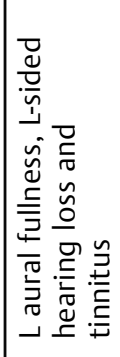 & 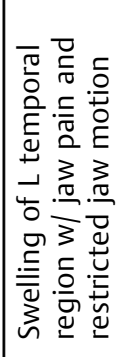 & 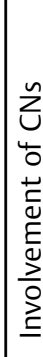 & 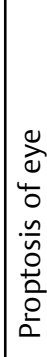 & 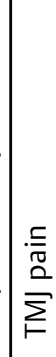 & 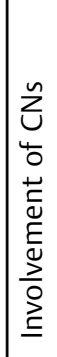 & 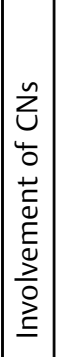 & 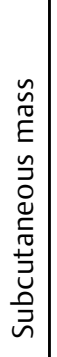 & 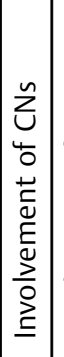 & 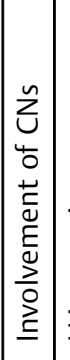 & 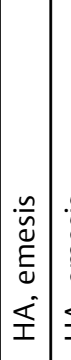 & 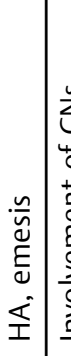 & 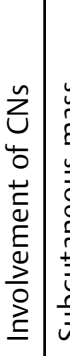 & 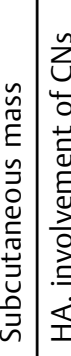 & 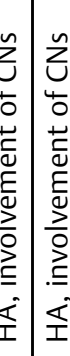 & 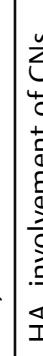 & \\
\hline 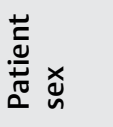 & $\Sigma$ & $\Sigma$ & $\Sigma$ & $\Sigma$ & $\Sigma$ & $\Sigma$ & $\Sigma$ & $\Sigma$ & $\Sigma$ & $\Sigma$ & $\Sigma$ & $\Sigma$ & 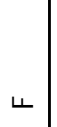 & 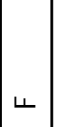 & $\Sigma$ & 4 & $\Sigma \Sigma$ & $\Sigma$ & \begin{tabular}{l|l} 
& 4
\end{tabular} & $\perp \mid \Sigma$ & $z$ & \\
\hline 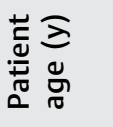 & 웅 & F & f & $\stackrel{m}{\sim}$ & $\stackrel{+}{m}$ & $\approx \tilde{m}$ & i̊ & ষ & $\neq$ & m & $\ddot{\sim}$ & $\stackrel{\infty}{\vdash}$ & 芒 & 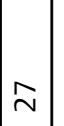 & a & 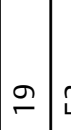 & กิ & $\stackrel{\sim}{\sim}$ & o & $\stackrel{\sim}{\sim} \stackrel{\stackrel{n}{N}}{ }$ & 운 & \\
\hline 层 & 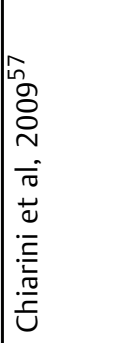 & 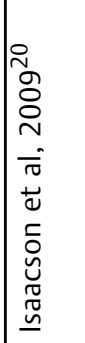 & & 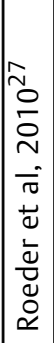 & 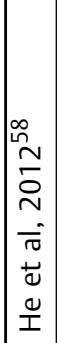 & 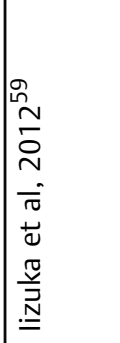 & 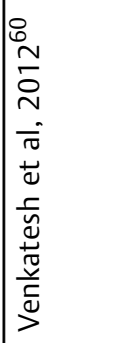 & 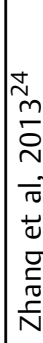 & & & & & & & & & & & & & & \\
\hline
\end{tabular}




\begin{tabular}{|c|c|c|c|c|c|c|c|c|c|c|c|c|}
\hline 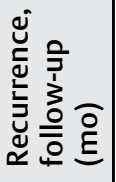 & $\Sigma$ & $\begin{array}{l}\simeq \\
z\end{array} \mid$ & $\begin{array}{l}0 \\
z\end{array}$ & $\begin{array}{l}n \\
z \\
z\end{array}$ & $\mid \begin{array}{l}\stackrel{一}{\simeq} \\
z ̇\end{array}$ & $\begin{array}{l}\infty \\
\stackrel{0}{0} \\
z ̇\end{array}$ & $\begin{array}{l}\text { \% } \\
z\end{array}$ & $\begin{array}{l}\stackrel{\infty}{+} \\
z\end{array}$ & $\begin{array}{l}\stackrel{\nabla}{\sim} \\
\succ\end{array}$ & $\begin{array}{l}\infty \\
\check{z} \\
z ̇\end{array}$ & $\begin{array}{l}\frac{\ln }{2} \\
z \ddot{z}\end{array}$ & $\begin{array}{l}0 \\
z\end{array}$ \\
\hline 崖 & $\S$ & $z$ & $z$ & $z$ & $z$ & $z$ & $z$ & $z$ & $>$ & $z$ & $z$ & $z$ \\
\hline 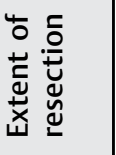 & $\stackrel{\frac{a}{\omega}}{a}$ & 兰 & 兰 & 鲁 & 总 & 总 & 关 & 兰 & $\frac{\underline{\alpha}}{\omega}$ & 兰 & 兰 & 㤐 \\
\hline 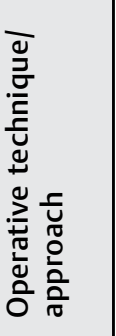 & $\Sigma$ & $\Sigma$ & $\S$ & 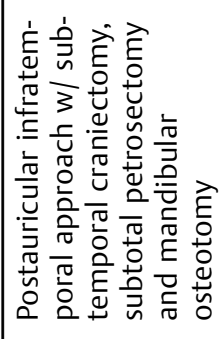 & 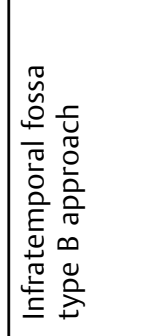 & 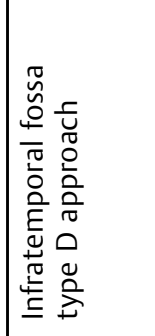 & 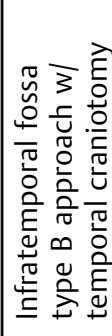 & 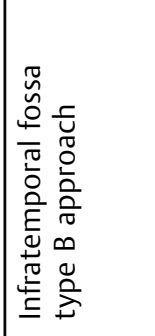 & 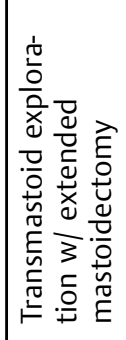 & 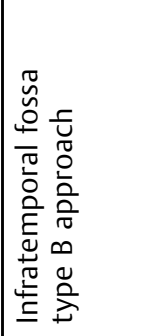 & 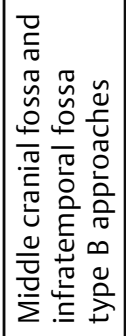 & 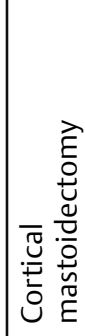 \\
\hline ᄃ。 & 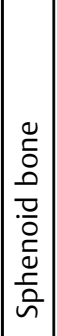 & 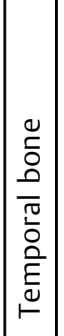 & 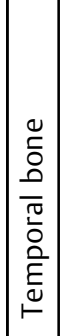 & 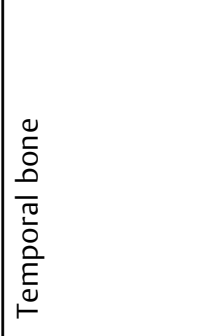 & 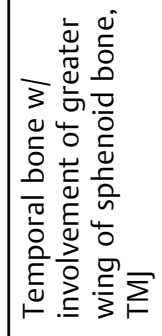 & 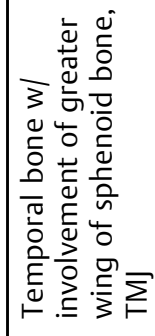 & 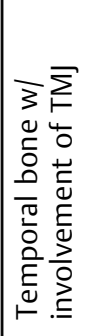 & 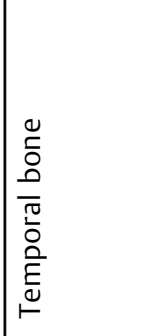 & 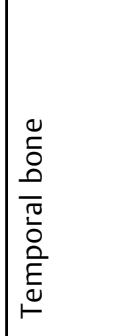 & 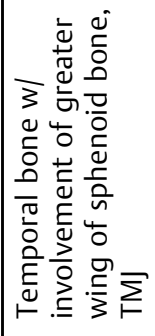 & 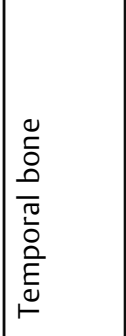 & 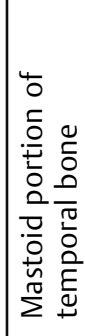 \\
\hline 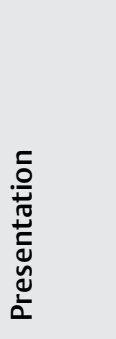 & 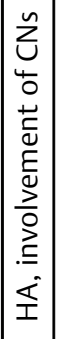 & 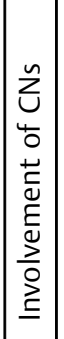 & 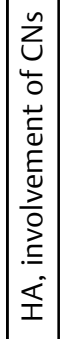 & 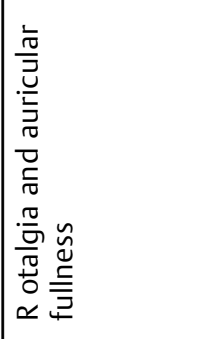 & 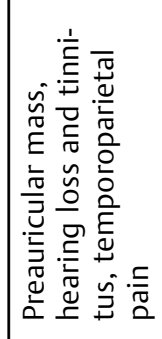 & 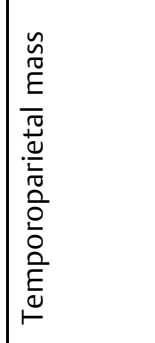 & 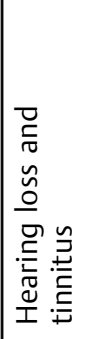 & 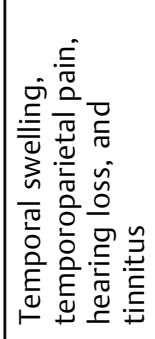 & 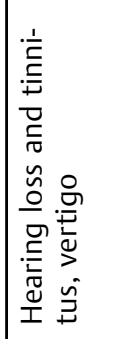 & 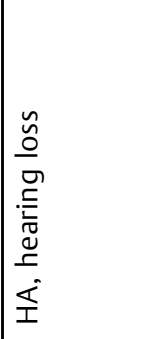 & 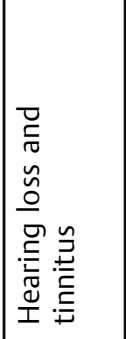 & 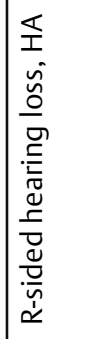 \\
\hline 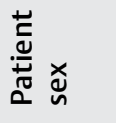 & 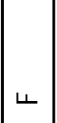 & $\Sigma$ & 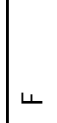 & 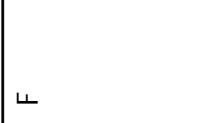 & $\Sigma$ & $\sqcup$ & 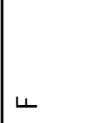 & $\Sigma$ & $\Sigma$ & $\Sigma$ & $\Sigma$ & $\Sigma$ \\
\hline 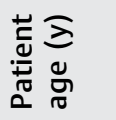 & $\tilde{m}$ & $m$ & $\stackrel{\stackrel{n}{m}}{m}$ & Ұ & $\stackrel{0}{m}$ & $\stackrel{\infty}{\sim}$ & $\bar{m}$ & 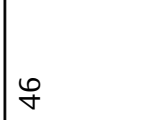 & 6 & શి & in & 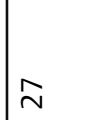 \\
\hline 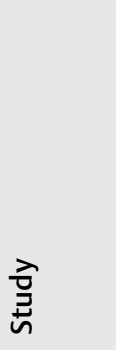 & & & & 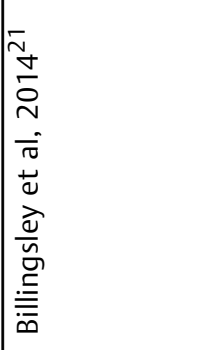 & 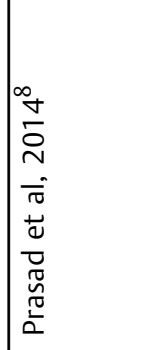 & & & & & & & 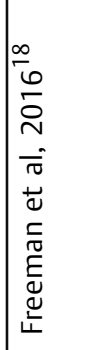 \\
\hline
\end{tabular}




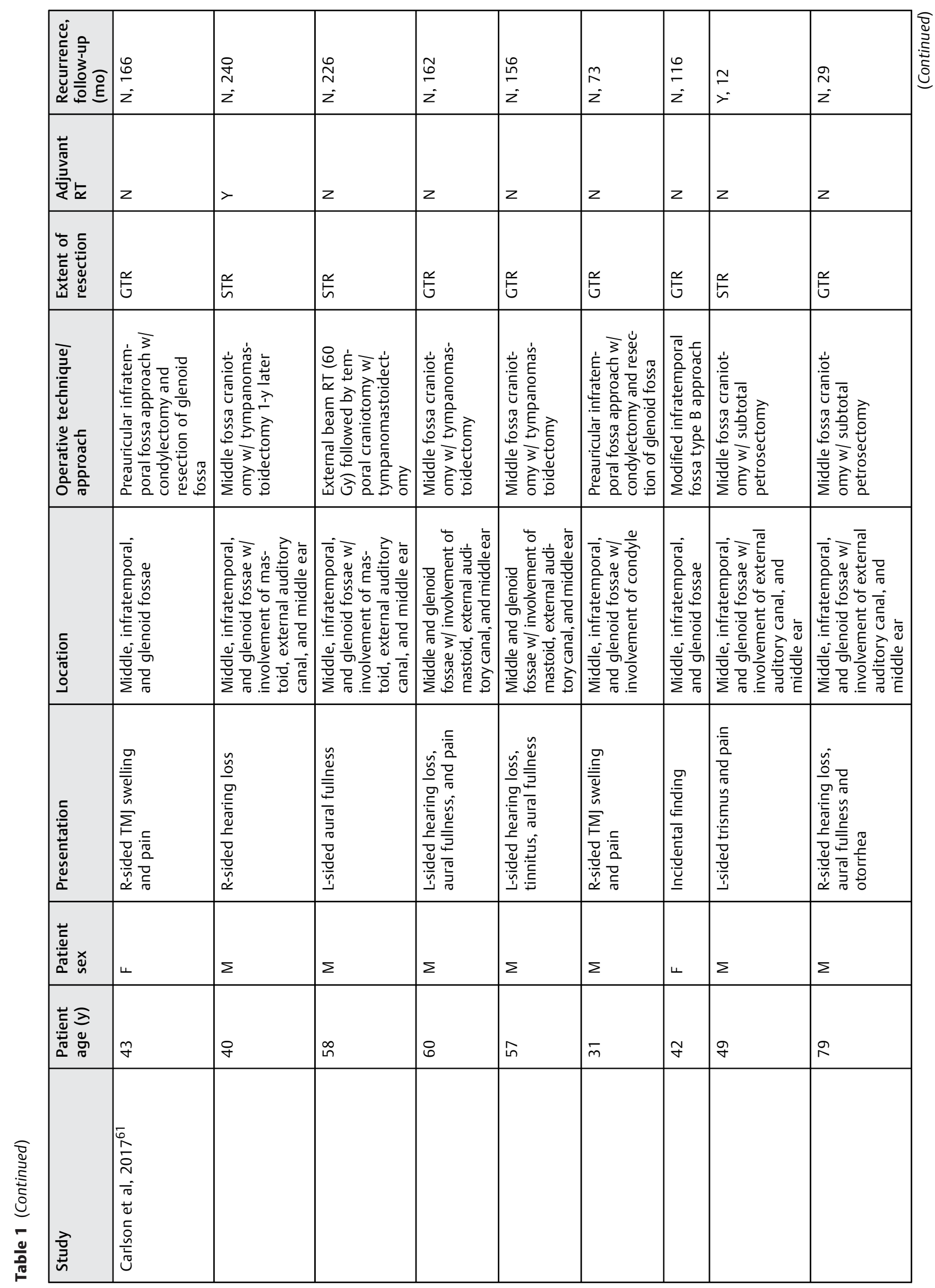




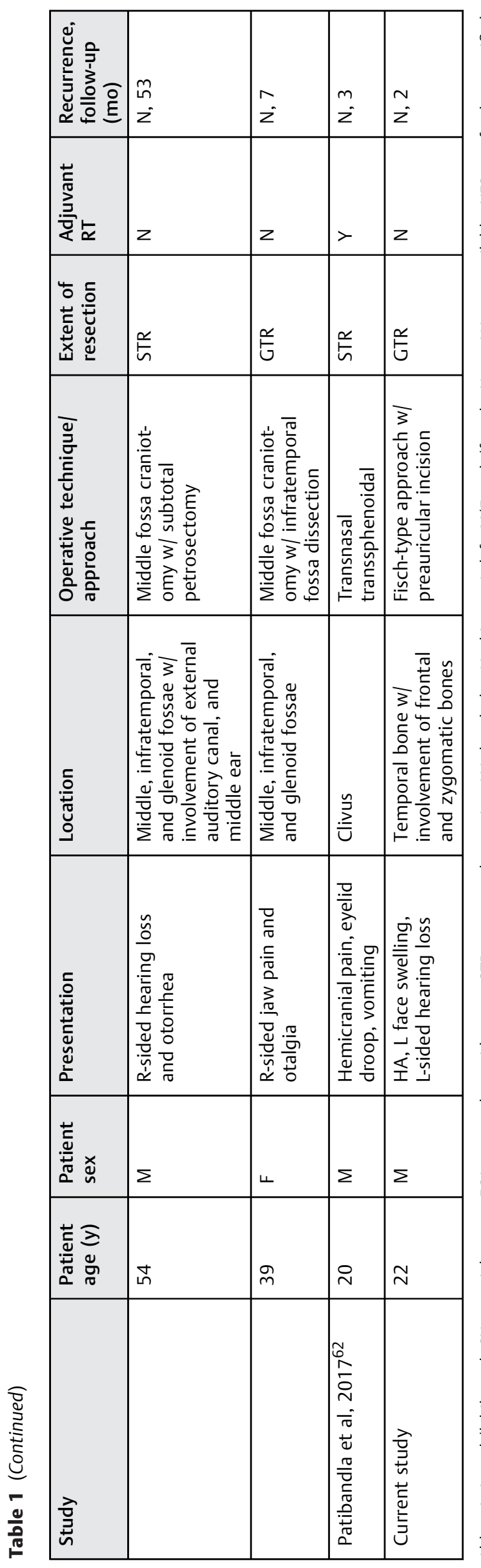

present as soft tissue masses of mixed density with higher density spots and destructive expansion into the bone, sometimes sparing cortical bone. ${ }^{12,18,24,25} \mathrm{CT}$ alone is insufficient for the accurate diagnosis and differentiation of GCTB from other similar-appearing masses or tumors, such as osteitis fibrosa cystica (brown tumor) or giant cell reparative granulomas. ${ }^{12,18}$ MRI provides better characterization and delineation of the tumor, showing intermediate signal intensity on T1-weighted imaging and hypointensity on T2weighted sequencing. ${ }^{18}$

Surgical removal of GCTB of the skull with complete resection is the current treatment of choice. ${ }^{8,14,18}$ The surgical management of GCTB of the lateral skull base can be difficult because of its proximity to critical neurovascular structures. Although gross total resection of the tumor is ideal, this may not be feasible depending on the extent of structural involvement by the tumor. In this setting, partial resection (i.e., maximal safe resection) followed by adjuvant radiotherapy may be a reasonable alternative. ${ }^{8,18,26,27} \mathrm{~A}$ recent systematic literature review of GCTBs involving the skull reported 94 patients who underwent surgery, with 37 of those patients having received adjuvant radiotherapy. ${ }^{24}$ In a subanalysis within this review, in 62 patients for whom survival data was available, the 5-year overall survival rate was $84 \%$, with an event-free survival rate (i.e., survival rate without tumor recurrence) of $61.3 \% .{ }^{24}$ All 16 patients who had gross total resection (with or without adjuvant radiotherapy) were alive and event-free at 5 years. ${ }^{24}$ In comparison, patients treated with subtotal resection and adjuvant radiotherapy ( $n=33$ ) had an overall survival of $90.3 \%$ and event-free survival of $70.1 \% .{ }^{24}$ Patients treated with subtotal resection without subsequent radiotherapy $(n=13)$ had an overall survival rate of $50 \%$ and an event-free rate of $15.4 \%$ at 5 years. $^{24}$ Because of the difficulty associated with the surgical management of GCTBs involving the lateral skull base, complications can arise during surgical resection (e.g., bleeding or the compromise of critical neural structures). Nevertheless, current evidence strongly suggests that gross total resection results in improved local control and survival outcomes. $^{18}$

Adjuvant therapy is recommended for cases where complete resection cannot be achieved. ${ }^{25}$ Radiation therapy is discussed in the literature as a possible treatment option following subtotal resection. Small retrospective studies and case reports have suggested a control benefit and marked symptom relief with adjuvant radiation therapy. Malone et $\mathrm{al}^{26}$ reported local control in 19 of 21 patients treated with radiation therapy, with a mean follow-up time of 15.4 years. Most patients received $35 \mathrm{~Gy}$ in 15 fractions delivered daily over 3 weeks. ${ }^{26}$ Other series have also demonstrated favorable local control rates, with Roeder et $\mathrm{al}^{27}$ reporting local control in four of five patients treated with intensitymodulated radiation therapy to a median dose of $64 \mathrm{~Gy}$ using conventional fractionation. There are also reports on the use of Gamma Knife (stereotactic) radiosurgery in the treatment of GCTB of the skull base, with marked reduction in tumor size and a meaningful disease-free interval. ${ }^{28}$ Although adjuvant radiation therapy appears to offer a control benefit, 
the data are limited by their retrospective nature, small patient numbers, and short follow-up intervals. Given these limitations, general consensus regarding optimal dose and fractionation regimens are lacking. Moreover, there is concern that radiotherapy may contribute to malignant transformation. ${ }^{20,25}$ This risk may be minimized by use of conventional fractionation regimens without compromising tumor control; however, longer follow-up is needed to better characterize this risk. ${ }^{15,25}$

Recently, several studies have investigated the role of targeted therapy with denosumab, a fully humanized monoclonal receptor activator of nuclear factor kappa-B ligand (RANK-L) antibody, which has shown promise as a potential effective chemotherapeutic treatment option in patients with GCTB. ${ }^{29}$ The osteoclastic giant cells responsible for local bony destruction and invasion in GCTB have been shown to ubiquitously express RANK receptor. ${ }^{29-31}$ Current evidence suggests that the neoplastic stromal cells promote the growth, proliferation, and osteolytic activity of the multinucleated osteoclastic giant cells through the overexpression of RANK-L, thus driving local bony destruction. ${ }^{30,32}$ With ongoing clinical trials studying the effectiveness of denosumab in the treatment of GCTBs, the role of monoclonal therapy in GCTBs of the skull base has not yet been fully characterized. ${ }^{31,33,34}$ Current evidence for the management of GCTBs of the skull recommends complete surgical resection with negative margins to achieve the highest rate of cure and lowest risk of recurrence. ${ }^{4,16}$ Nevertheless, the inhibition of this pathway with a monoclonal RANK-L antibody may limit bony destruction and tumor progression, thereby making these tumors of the lateral skull base more amenable to complete surgical resection and therefore decreasing the morbidity and mortality of this disease.

\section{Conclusion}

The skull is a relatively rare location for a GCTB to occur. When this tumor is found, it is normally found in the temporal or sphenoid bones. Although GCTB is a benign tumor, it is also locally aggressive and has the ability to recur or rarely metastasize. Gross total resection is the current treatment of choice in GCTB of the skull, but it can be difficult to achieve due to the proximity of the tumor to important neural and vascular structures. Subtotal resection with adjuvant radiotherapy may be a good alternative treatment in such cases. Although radiotherapy has been the adjuvant therapy of choice in GCTB treatment, recent literature shows that denosumab, a RANK-L antibody, may also prove to be effective in treating the tumor. Future studies should evaluate the efficacy of different adjuvant therapies used to treat partially resected GCTB of the lateral skull base.

\section{Disclosure}

None.

\section{Compliance with Ethical Standards}

This study was approved by the Institutional Review

Board (IRB) of the University of Utah.

\section{Patient Consent}

The patient/legal guardian/next of kin has consented to the submission of the case report for submission to the Journal of Neurological Surgery Reports.

\section{Acknowledgment}

The authors thank Kristin Kraus, M.Sc., for her assistance with preparation of this article.

\section{References}

1 Larsson SE, Lorentzon R, Boquist L. Giant-cell tumor of bone. A demographic, clinical, and histopathological study of all cases recorded in the Swedish Cancer Registry for the years 1958 through 1968. J Bone Joint Surg Am 1975;57(02):167-173

2 Baena-Ocampo Ldel C, Ramirez-Perez E, Linares-Gonzalez LM, Delgado-Chavez R. Epidemiology of bone tumors in Mexico City: retrospective clinicopathologic study of 566 patients at a referral institution. Ann Diagn Pathol 2009;13(01):16-2

3 Beebe-Dimmer JL, Cetin K, Fryzek JP, Schuetze SM, Schwartz K. The epidemiology of malignant giant cell tumors of bone: an analysis of data from the Surveillance, Epidemiology and End Results Program (1975-2004). Rare Tumors 2009;1(02):e52. Doi: 10.4081/rt.2009.e52

4 Campanacci M, Baldini N, Boriani S, Sudanese A. Giant-cell tumor of bone. J Bone Joint Surg Am 1987;69(01):106-114

5 Wang Y, Honda K, Suzuki S, Ishikawa K. Giant cell tumor at the lateral skull base. Am J Otolaryngol 2006;27(01):64-67

6 Werner M. Giant cell tumour of bone: morphological, biological and histogenetical aspects. Int Orthop 2006;30(06):484-489

7 Mendenhall WM, Zlotecki RA, Scarborough MT, Gibbs CP, Mendenhall NP. Giant cell tumor of bone. Am J Clin Oncol 2006;29(01):96-99

8 Prasad SC, Piccirillo E, Nuseir A, et al. Giant cell tumors of the skull base: case series and current concepts. Audiol Neurootol 2014;19 (01):12-21

9 Qureshi SS, Puri A, Agarwal M, Desai S, Jambhekar N. Recurrent giant cell tumor of bone with simultaneous regional lymph node and pulmonary metastases. Skeletal Radiol 2005;34(04):225-228

10 Balke M, Schremper L, Gebert C, et al. Giant cell tumor of bone: treatment and outcome of 214 cases. J Cancer Res Clin Oncol 2008;134(09):969-978

11 Dahlin DC, Cupps RE, Johnson EW Jr. Giant-cell tumor: a study of 195 cases. Cancer 1970;25(05):1061-1070

12 Lee HJ, Lum C. Giant-cell tumor of the skull base. Neuroradiology 1999;41(04):305-307

13 Saleh EA, Taibah AK, Naguib M, et al. Giant cell tumor of the lateral skull base: a case report. Otolaryngol Head Neck Surg 1994;111(3 Pt 1):314-318

14 Shen Y, Ma C, Wang L, Li J, Wu Y, Sun J. Surgical management of giant cell tumors in temporomandibular joint region involving lateral skull base: a multidisciplinary approach. J Oral Maxillofac Surg 2016;74(11):2295-2311

15 Bertoni F, Unni KK, Beabout JW, Ebersold MJ. Giant cell tumor of the skull. Cancer 1992;70(05):1124-1132

16 Klenke FM, Wenger DE, Inwards CY, Rose PS, Sim FH. Giant cell tumor of bone: risk factors for recurrence. Clin Orthop Relat Res 2011;469(02):591-599

17 Wong RH, Thakral B, Watkin WG, Merrell R, Wong AK, Farhat HI. Intracranial, intra-axial metastatic giant cell tumor of bone: case report and review of literature. Clin Neurol Neurosurg 2014; 117:40-43

18 Freeman JL, Oushy S, Schowinsky J, Sillau S, Youssef AS. Invasive giant cell tumor of the lateral skull base: a systematic review, metaanalysis, and case illustration. World Neurosurg 2016;96:47-57

19 Wolfe JT III, Scheithauer BW, Dahlin DC. Giant-cell tumor of the sphenoid bone. Review of 10 cases. J Neurosurg 1983;59(02):322-327 
20 Isaacson B, Berryhill W, Arts HA. Giant-cell tumors of the temporal bone: management strategies. Skull Base 2009;19(04): 291-301

21 Billingsley JT, Wiet RM, Petruzzelli GJ, Byrne R. A locally invasive giant cell tumor of the skull base: case report. J Neurol Surg Rep 2014;75(01):e175-e179

22 Morriss-Kay GM. Derivation of the mammalian skull vault. J Anat 2001;199(Pt 1-2):143-151

23 Findlay JM, Chiasson D, Hudson AR, Chui M. Giant-cell tumor of the middle cranial fossa. Case report. J Neurosurg 1987;66(06): 924-928

24 Zhang Z, Xu J, Yao Y, et al. Giant cell tumors of the skull: a series of 18 cases and review of the literature. J Neurooncol 2013;115(03): 437-444

25 Sharma RR, Mahapatra AK, Pawar SJ, Sousa J, Dev EJ. Craniospinal giant cell tumors: clinicoradiological analysis in a series of 11 cases. J Clin Neurosci 2002;9(01):41-50

26 Malone S, O'Sullivan B, Catton C, Bell R, Fornasier V, Davis A. Longterm follow-up of efficacy and safety of megavoltage radiotherapy in high-risk giant cell tumors of bone. Int J Radiat Oncol Biol Phys 1995;33(03):689-694

27 Roeder F, Timke C, Zwicker F, et al. Intensity modulated radiotherapy (IMRT) in benign giant cell tumors-a single institution case series and a short review of the literature. Radiat Oncol 2010; 5:18. Doi: 10.1186/1748-717X-5-18

28 Kim IY, Jung S, Jung TY, et al. Gamma knife radiosurgery for giant cell tumor of the petrous bone. Clin Neurol Neurosurg 2012;114 (02):185-189

29 Xu SF, Adams B, Yu XC, Xu M. Denosumab and giant cell tumour of bone-a review and future management considerations. Curr Oncol 2013;20(05):e442-e447

30 Huang L, Xu J, Wood DJ, Zheng MH. Gene expression of osteoprotegerin ligand, osteoprotegerin, and receptor activator of NFkappaB in giant cell tumor of bone: possible involvement in tumor cell-induced osteoclast-like cell formation. Am J Pathol 2000;156(03):761-767

31 Thomas DM. RANKL, denosumab, and giant cell tumor of bone. Curr Opin Oncol 2012;24(04):397-403

32 López-Pousa A, Martín Broto J, Garrido T, Vázquez J. Giant cell tumour of bone: new treatments in development. Clin Transl Oncol 2015;17(06):419-430

33 Chawla S, Henshaw R, Seeger L, et al. Safety and efficacy of denosumab for adults and skeletally mature adolescents with giant cell tumour of bone: interim analysis of an open-label, parallel-group, phase 2 study. Lancet Oncol 2013;14(09): 901-908

34 Thomas D, Henshaw R, Skubitz K, et al. Denosumab in patients with giant-cell tumour of bone: an open-label, phase 2 study. Lancet Oncol 2010;11(03):275-280

35 Carmody RF, Rickles DJ, Johnson SF. Giant cell tumor of the sphenoid bone. J Comput Assist Tomogr 1983;7(02):370-373

36 Motomochi M, Handa Y, Makita Y, Hashi K. Giant cell tumor of the skull. Surg Neurol 1985;23(01):25-30

37 Kiwit JCW, Schober R, Nicola N, Schirmer M, Wechsler W. Osteoclastomas of the petrous bone. Surg Neurol 1986;26(01): 59-62

38 Tandon DA, Deka RC, Chaudhary C, Misra NK. Giant cell tumour of the temporosphenoidal region. J Laryngol Otol 1988;102(05): 449-451

39 do Amaral CMR, Julio GL, Cardoso LAA, Bueno Mde C. Craniofacial treatment of giant-cell tumors of the sphenoid bone. J Craniofac Surg 1994;5(04):254-256

40 Rock JP, Mahmood A, Cramer HB. Giant cell tumor of the skull base. Am J Otol 1994;15(02):268-272
41 Silvers AR, Som PM, Brandwein M, Chong JL, Shah D. The role of imaging in the diagnosis of giant cell tumor of the skull base. AJNR Am J Neuroradiol 1996;17(07):1392-1395

42 Büter JJ, Chilla R. Giant cell tumor of the temporal bone (osteoclastoma). Eur Arch Otorhinolaryngol 1997;254(06):298-300

43 Li KK, Ung F, McKenna MJ, Keith DA. Combined middle cranial fossa and preauricular approach to the temporomandibular joint: report of a case. J Oral Maxillofac Surg 1997;55(08):851-852

44 Kattner KA, Stroink A, Gupta K, Fukushima T, Li C. Giant cell tumor of the sphenoid bone. Skull Base Surg 1998;8(02):93-97

45 Omura S, Mizuki N, Bukawa H, Fujita K. Diffuse variant tenosynovial giant cell tumor of the temporomandibular joint: report of a case. J Oral Maxillofac Surg 1998;56(08):991-996

46 Rosenbloom JS, Storper IS, Aviv JE, Hacein-Bey L, Bruce JN. Giant cell tumors of the jugular foramen. Am J Otolaryngol 1999;20(03): 176-179

47 Spallone A, Flores GL, Zaldivar LO, Estupinan B. Giant cell tumor (osteoclastoma) of the petrous bone: case report. Skull Base Surg 1999;9(02):155-159

48 Bibas-Bonet H, Fauze RA, Lavado MG, Páez RO, Nieman J. Garcin syndrome resulting from a giant cell tumor of the skull base in a child. Pediatr Neurol 2003;28(05):392-395

49 Chan J, Gannon FH, Thompson LDR. Malignant giant cell tumor of the sphenoid. Ann Diagn Pathol 2003;7(02):100-105

50 Harris AE, Beckner ME, Barnes L, Kassam A, Horowitz M. Giant cell tumor of the skull: a case report and review of the literature. Surg Neurol 2004;61(03):274-277

51 Tang JY, Wang CK, Su YC, Yang SF, Huang MY, Huang CJ. MRI appearance of giant cell tumor of the lateral skull base: a case report. Clin Imaging 2003;27(01):27-30

52 Pai SB, Lalitha RM, Prasad K, Rao SG, Harish K. Giant cell tumor of the temporal bone-a case report. BMC Ear Nose Throat Disord 2005;5:8. Doi: 10.1186/1472-6815-5-8

53 Zorlu F, Selek U, Soylemezoglu F, Oge K. Malignant giant cell tumor of the skull base originating from clivus and sphenoid bone. J Neurooncol 2006;76(02):149-152

54 Elder JB, Berry C, Gonzalez-Gomez I, Kreger MD, McComb JG. Giant cell tumor of the skull in pediatric patients. Report of two cases. J Neurosurg 2007;107(1, Suppl):69-74

55 Gupta R, Mohindra S, Mahore A, Mathuriya SN, Radotra BD. Giant cell tumour of the clivus. Br J Neurosurg 2008;22(03):447-449

56 Matsushige T, Nakaoka M, Yahara K, et al. Giant cell tumor of the temporal bone with intratumoral hemorrhage. J Clin Neurosci 2008;15(08):923-927

57 Chiarini L, Figurelli S, Ghidini A, et al. An unusual case of giant cell tumour involving the middle cranial fossa, originating from soft tissues of the temporomandibular joint. Acta Neurochir (Wien) 2009;151(12):1685-1687

58 He D, Yang C, Shen G, et al. Navigation-guided resection for a tenosynovial giant cell tumor involving the temporomandibular joint and skull base. J Craniofac Surg 2012;23(02):521-523

59 Iizuka T, Furukawa M, Ishii H, et al. Giant cell tumor of the temporal bone with direct invasion into the middle ear and skull base: a case report. Case Rep Otolaryngol 2012;2012:690148

60 Venkatesh MD, Vijaya N, Girish N, Galagali JR. Giant cell tumor of temporal bone: a case report. Med J Armed Forces India 2012;68 (04):392-394

61 Carlson ML, Osetinsky LM, Alon EE, Inwards CY, Lane JI, Moore EJ Tenosynovial giant cell tumors of the temporomandibular joint and lateral skull base: review of 11 cases. Laryngoscope 2017;127 (10):2340-2346

62 Patibandla MR, Thotakura AK, Rao MN, et al. Clival giant cell tumor a rare case report and review of literature with respect to current line of management. Asian J Neurosurg 2017;12(01):78-81 